\title{
Tensor-Based Noninvasive Atrial Fibrillation Complexity Index For Catheter Ablation
}

\author{
Lucas de S Abdalah ${ }^{1,2}$, Pedro Marinho R de Oliveira ${ }^{1}$, Walter Freitas $\mathrm{Jr}^{2}$, Vicente Zarzoso ${ }^{1}$ \\ ${ }^{1}$ Université Côte d'Azur, CNRS, I3S Laboratory, Sophia Antipolis, France \\ ${ }^{2}$ Universidade Federal do Ceará, Fortaleza, Brazil
}

\begin{abstract}
Due to the cost-efficiency of the ECG, the interest in noninvasive techniques to assess atrial fibrillation $(A F)$ electrophysiological complexity is increasingly high. Still, ECG-based methods to measure AF complexity are limited in clinical practice and need estimation of the atrial activity (AA) signal from sufficiently long ECG recordings. The present work proposes an algorithm for tensor decomposition called constrained alternating group lasso (CAGL) as a noninvasive tool to quantify $A F$ complexity. Experiments with a database of 59 ECG recordings from 20 patients suffering from persistent AF show that CAGL is able to both extract the $A A$ and quantify its complexity from very short ECG recordings $(1.06 \pm 0.20 \mathrm{~s})$. All the patients had undergone step-wise catheter ablation (CA) that ended in procedural AF termination. CAGL is applied on the ECG recording before $C A$ and at each step procedure, measuring the rank of the tensor that provides the AA signal. It is observed that such rank decreases at each step of the $C A$ procedure, showing a less complex AA signal as the $a b-$ lation is performed. A statistical correlation between $A A$ complexity measured by the new index and $A F$ recurrence after CA is observed. The proposed index is a potential tool to guide CA procedures in real time.
\end{abstract}

\section{Introduction}

Atrial fibrillation (AF) is the most frequent sustained arrhythmia encountered in clinical practice, responsible for an increasingly high number of hospitalizations and deaths [1]. This challenging cardiac condition is known as the last great frontier in cardiac electrophysiology, as the electrophysiological mechanisms responsible for its triggering and maintenance are not completely understood. A noninvasive and cost-effective way to study this cardiac rhythm disorder is analyzing the atrial activity (AA) signal from the standard 12-lead electrocardiogram (ECG). However, the AA during AF is characterized by low-amplitude fibrillatory waves, called f-waves, that are masked by the
QRS complex responsible for the ventricular activity (VA) in each heartbeat. In addition, the AA sometimes presents an amplitude lower than the noise, hampering its analysis [2].

During AF, the AA and the VA signals are typically assumed uncoupled, so that the extraction of AA from the ECG admits a blind source separation (BSS) formulation [3]. The block-term decomposition (BTD) built from Hankel matrices, proposed as a technique to solve BSS problems in [4], was used to noninvasively extract the AA signal from AF ECG recordings, showing that it can outperform the matrix-based techniques in this particular application [5], [6].

Step-wise catheter ablation (CA) is an effective therapy to treat persistent $\mathrm{AF}$ and restore sinus rhythm [7]. Hence, methods to measure AA complexity at each procedural step are relevant to improve clinical analysis and guide $\mathrm{CA}$ in real time. Furthermore, it would be desirable to clarify the impact on AF of each intervention step such as pulmonary vein isolation (PVI) and other widely used techniques [8], [9]. However, existing methods for noninvasive quantification of $\mathrm{AF}$ complexity are limited due to the fact that sufficiently long ECG recordings are required, hindering their use in clinical practice.

Aiming to overcome such limitations, the present work introduces a recently proposed algorithm to compute the BTD, called CAGL [10], as an AF complexity index. CAGL is able to jointly extract the AA signal from the ECG and measure AF complexity from very short recordings $(1.06 \pm 0.20 \mathrm{~s})$. The AF complexity is measured from the rank of the tensor block associated with the AA signal.

\section{Methods}

\subsection{Block Term Decomposition}

An ECG recording from $K$ leads composed by $N$ time samples can be modeled as a matrix factorization:

$$
\mathbf{Y}=\mathbf{M S} \in \mathbb{R}^{K \times N}
$$

where $\mathbf{M} \in \mathbb{R}^{K \times R}$ is the mixing matrix, modeling the propagation of the cardiac electrical sources from the heart 
to the body surface, $\mathbf{S} \in \mathbb{R}^{R \times N}$ is the source matrix that contains mainly the atrial and ventricular sources and $R$ is the number of sources [3]. Nevertheless, matrix-based methods impose constraints that may lack physiological grounds. In this case, we propose a tensor approach using Hankel-BTD model that outperform matrix-based techniques under much milder constraints [4], [10].

A tensor is an extension of the matrix concept. While matrices are two-dimensional arrays, a third-order tensor $\mathcal{Y} \in \mathbb{R}^{I \times J \times K}$ is a three-dimensional array, whose BTD is written as:

$$
\mathcal{Y}=\sum_{r=1}^{R}\left(\mathbf{A}_{\mathbf{r}} \mathbf{B}_{\mathbf{r}}^{\top}\right) \circ \mathbf{x}_{\mathbf{r}}
$$

where $\mathbf{A}_{r} \in \mathbb{R}^{I \times L_{r}}$ and $\mathbf{B}_{r} \in \mathbb{R}^{J \times L_{r}}$ are the factors matrices, and have rank $L_{r}$. The symbols $(\cdot)^{\top}$ and o denote the transposition operator, and the outer product, respectively.

The Hankel-BTD method suits the characteristics of AA in AF episodes. Due to the quasi-periodic nature of AF signals, atrial sources can be well represented by an all-pole model, i.e., a sum of complex exponentials [11]. Hence, each ECG lead can be mapped onto a Hankel matrix, and these matrices are stacked in a third-order tensor $\mathcal{Y}_{\mathcal{H}}$, as described in [4], [6], that admits the following model:

$$
\mathcal{Y}_{\mathcal{H}}=\sum_{r=1}^{R} \mathbf{H}_{\mathbf{S}}^{(r)} \circ \mathbf{m}_{. r}
$$

where $\mathbf{H}_{\mathbf{S}}^{(r)}$ is a Hankel matrix built from the $r^{\text {th }}$ source of $\mathbf{S}$, and $\mathbf{m}_{. r}$ is the $r^{\text {th }}$ mixing matrix column.

\subsection{Constrained Alternating Group Lasso}

In general, an approximate BTD is computed by minimizing the Euclidean distance between the observed data tensor $\mathcal{Y} \in \mathbb{C}^{\mathbb{I} \times \mathbb{J} \times \mathbb{K}}$ and a model of fixed structure with respect to the model components:

$$
f(\mathbf{A}, \mathbf{B}, \mathbf{X}) \triangleq\left\|\mathcal{Y}-\sum_{\mathbf{r}=1}^{\mathbf{R}}\left(\mathbf{A}_{\mathbf{r}} \mathbf{B}_{\mathbf{r}}^{\top}\right) \circ \mathbf{x}_{\mathbf{r}}\right\|_{\mathbf{F}}^{2} .
$$

In the special case of interest, $\mathbf{H}_{\mathbf{r}}$ must belong to the subspace of Hankel matrices with dimensions $(I \times J)$, denoted $\mathcal{S}_{\mathcal{H}}$. The mode-3 slices $\mathbf{Y}_{\text {..k }}$ of the observed tensor are Hankel by construction. However, a solution $(\hat{\mathbf{A}}, \hat{\mathbf{B}}, \hat{\mathbf{X}})$ of (4) may not satisfy $\hat{\mathbf{A}}_{r} \hat{\mathbf{B}}_{r}^{T} \in \mathcal{S}_{\mathcal{H}}$, due to noise and modeling imperfections. Also, algorithms based on (4) are strongly dependent of the initialization of its matrix factors and do not estimate the model parameters, i.e., the number of blocks and their ranks.

To overcome such limitations, instead of using a fixed BTD structure as in (4), an algorithm called AGL and its constrained version described for Hankel matrices called
CAGL are proposed in [10]. This method includes penalization terms promoting low-rank blocks and controlling the number of blocks, yielding criteria of the form:

$$
F(\mathbf{A}, \mathbf{B}, \mathbf{X}) \triangleq f(\mathbf{A}, \mathbf{B}, \mathbf{X})+\gamma g(\mathbf{A}, \mathbf{B}, \mathbf{X})
$$

where $\gamma>0$ is a regularization parameter and $g$ is a regularization function of the form:

$$
g(\mathbf{A}, \mathbf{B}, \mathbf{X}) \triangleq\|\mathbf{A}\|_{2,1}+\|\mathbf{B}\|_{2,1}+\|\mathbf{X}\|_{2,1} .
$$

Notation $\|\cdot\|_{2,1}$ denotes the mixed $\ell_{2,1}$-norm, i.e., the sum of $\ell_{2}$ norms of the columns of its matrix argument. Due to the geometric properties of the mixed $\ell_{2,1}$-norm, solutions where $\mathbf{A}, \mathbf{B}$ and $\mathbf{X}$ have null columns (for sufficiently high $\gamma$ values) will be induced, allowing one to select the relevant low-rank blocks. This method is called group lasso and is a generalization of the the lasso estimator principle [12].

In order to ensure the Hankel structure of the matrix factors at the end of iterations, a structured low-rank approximation is applied at convergence of the algorithm, yielding CAGL. For this purpose, the Cadzow's Algorithm [13] is used at the end of the iterations, which consists in performing alternating projections onto the Hankel subspace $\mathcal{S}_{\mathcal{H}}$, so that $\hat{\mathbf{H}}_{r} \approx \hat{\mathbf{A}}_{r} \hat{\mathbf{B}}_{r}^{T} \in \mathcal{S}_{\mathcal{H}}$. A detailed description of the CAGL algorithm can be found in [10].

\subsection{Tensor-Based AF Complexity Index}

The complexity of a signal constructed from complex exponential sums is intrinsically related to the number of poles. Since the tensor block correlated with the AA signal presents a Hankel structure, the rank of its matrix factor $L_{r}$ is equal to the number of poles [5]. Therefore, the proposed index is measured from the rank of the block that represents the atrial source, that allows a more global view of what goes on in the atria, while a catheter provides more local information.

\subsection{Database and Experimental Setup}

The present database consists in 59 ECG recordings from 20 patients suffering from persistent AF, who had undergone step-wise CA that ended in procedural AF termination. All recordings belong to the Cardiology Department of Princess Grace Hospital Center, Monaco, acquired at a $977 \mathrm{~Hz}$ sampling rate. They are preprocessed by a zero-phase forward-backward type-II Chebyshev bandpass filter with cutoff frequencies of 0.5 and $40 \mathrm{~Hz}$, in order to suppress high-frequency noise and baseline wandering.

Twenty male patients compose the population with average age, height and weight, respectively, of $60.6 \pm 9.4$ years, $177.8 \pm 6.2 \mathrm{~cm}$ and $85.6 \pm 12.7 \mathrm{~kg}$. Left atrium ${ }^{1}$

\footnotetext{
${ }^{1}$ The value for one patient was replaced by the mean.
} 

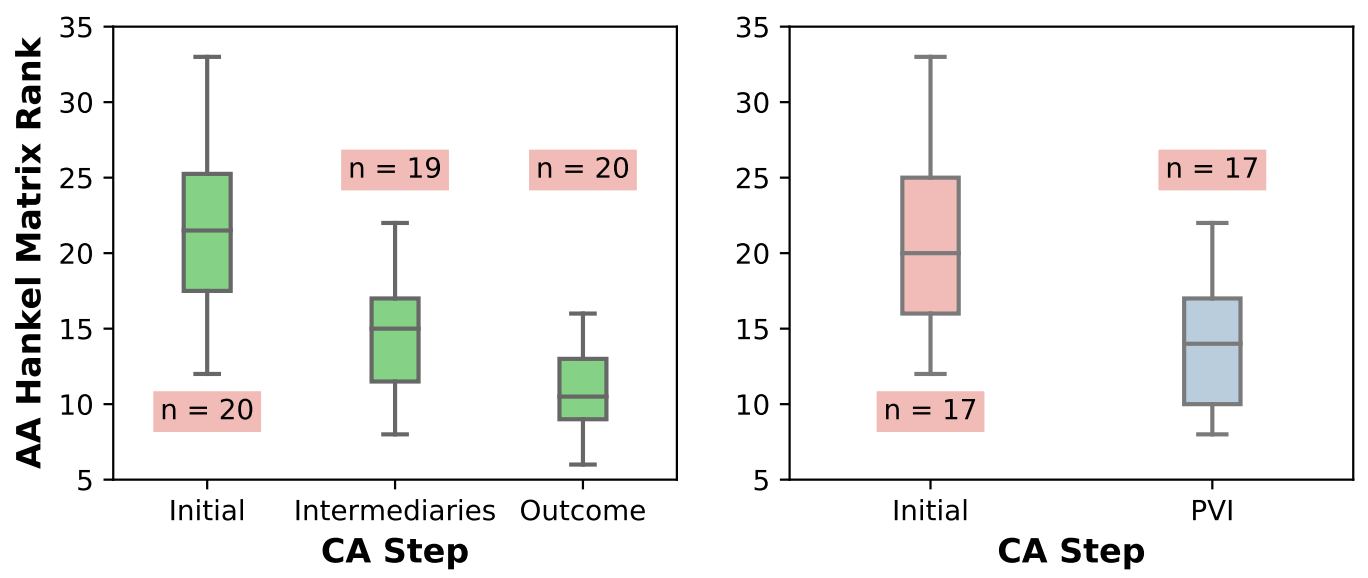

Figure 1. Right: Box-and-whisker plot showing the rank estimated by CAGL for all patients at different CA steps: initial (before ablation); intermediaries (CA between the first and penultimate steps); outcome (after the last CA step). Left: Boxand-whisker plot at the beginning of CA and after PVI for the group of 17 patients who underwent this CA step. Notation (n) indicates the number of ECG segments considered in each box.

diameter was $45.8 \pm 7.9 \mathrm{~mm}$. In addition, AF history was of $68.6 \pm 59.6$ months, while duration of the current AF episode (ongoing at the time of CA) was $16.3 \pm 25.2$ months.

The segment with the largest TQ segment is chosen for each patient, length ranging from 0.72 to 1.42 seconds. A window with length $1.06 \mathrm{~s}$, yields 1037 samples, a direct row-Hankelization of this matrix results in a tensor of dimensions $519 \times 519 \times 12$, whose approximate BTD demands a large computing time. Therefore, we downsample the signals by a factor of 10 before apply the decomposition in order to reduce its computing time, with practically negligible information loss. In this example, the resulting tensor $\mathcal{Y}$ have dimensions $52 \times 53 \times 12$.

CAGL is applied to ECG recordings after each CA step, with a $\gamma$-sweeping procedure, inspired by solution-path techniques, by taking 50 equispaced values in the interval $\left[8 \times 10^{-4}, 0.5 \times 10^{-2}\right]$ and keeping the last solution. We start the algorithm with $R=6$ random blocks and rank $L_{r}=40$ as initial guess [10]. The task of measuring estimation quality is challenging since the ground truth is unknown. Nevertheless some AA characteristics during AF must be exploited to guide sources selection. The parameters used to evaluate AA extraction are spectral concentration (SC), dominant frequency (DF) and kurtosis as well as visual inspection, as detailed in [6], [14], [15].

\section{Experimental Results}

\subsection{AA Complexity influence by PVI}

The impact of CA at each step on AA complexity is assessed in terms of rank estimation by CAGL in the whole dataset. Before $\mathrm{CA}$, ranks range from 12 to 33, whereas after all steps of the CA procedure, ranks range from 6 to 16 , referred to, respectively, as 'Initial' and 'Outcome' in Figure 1. Initially, the population present a median rank 21.5, while at intermediaries steps is 15 and after CA it becomes 10.5, illustrating that the rank decreases at each step of the CA procedure as shown in Figure 1. This plot graphically depicts data through their quartiles, with boxes' edges represent the 25 th and 75 th percentiles, horizontal line in the middle of the box represents sample median and black whiskers represent the extreme data values that are not considered as outliers.

In observed segments, the rank of extracted AA sources becomes less complex as the ablation is performed as one could intuitively expect. In addition, a separate assessment of the 17 patients undergoing PVI is also shown in Figure 1, presenting a drastic reduction of the proposed index after this CA step, from a median value of 20 to 14 .

\subsection{AF Recurrence and Complexity}

To assess the relationship between population's features, Pearson correlation $(r)$ between the initial rank estimated by CAGL, i.e., before any patient had undergone CA procedure, and $\mathrm{AF}$ recurrence, i.e., the time that each patient remained in sinus rhythm before AF relapse is -0.63 . A statistical relevant value of negative correlation well illustrated by its scatter plot and linear regression in Figure 2, seems to indicate an influence of initial rank on AF recurrence.

Finally, 2 patients registered ranks 12 and 18 but had no information about $\mathrm{AF}$ recurrence after the $\mathrm{CA}$ procedure, since they dropped out of the study. Hence, they were excluded from this assessment. 


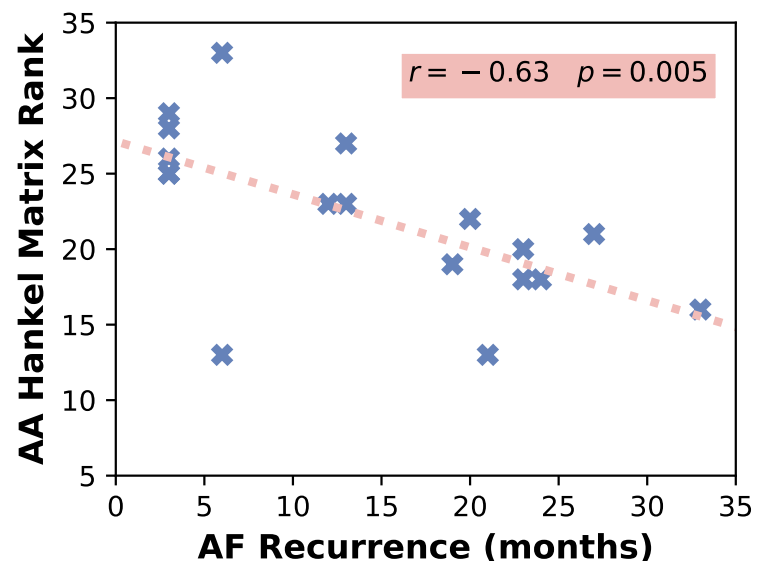

Figure 2. Scatter plot of the initial estimated rank (before $\mathrm{CA}$ ) of the tensor block that provides the AA signal versus AF recurrence. A negative correlation can be observed.

\section{Conclusions}

The present work proposed a novel index to noninvasively measure the AF complexity using tensor models. This index is based on the CAGL algorithm for BTD computation, which is able to jointly extract the AA signal and measure AF complexity from very short ECG recordings. Experiments on a database of $20 \mathrm{AF}$ patients have shown that the rank of the block that provides the AA signal decreases at each step of the CA procedure. Also, it was observed a negative correlation between the estimated rank and $\mathrm{AF}$ recurrence. In conclusion, this rank parameter could improve clinical analysis and support real-time guided CA, improving its accuracy, while reducing its cost and duration.

Future work should focus on comparing the proposed complexity parameter with other state-of-the-art indices and performing experiments in a larger database of patients in order to provide more relevant clinical results.

\section{Acknowledgments}

Lucas de S. Abdalah is supported by the BRAFITEC program at Université Côte d'Azur, financed by CAPES within the Ministry of Education of Brazil. Pedro Marinho R. de Oliveira is funded by a PhD scholarship from the IT Doctoral School (ED STIC) of the Université Côte d'Azur.

\section{References}

[1] C. T. January, L. S. Wann, H. Calkins, J. E. Cigarroa, et al., "2019 AHA/ACC/HRS focused update of the 2014 AHA/ACC/HRS guideline for the management of patients with atrial fibrillation," Journal of the American College of Cardiology, vol. 74, no. 1, pp. 104-132, 2019.
[2] L. Mainardi, L. Sörnmo, and S. Cerutti, Understanding Atrial Fibrillation: The Signal Processing Contribution. Morgan \& Claypool Publishers, 2008.

[3] J. J. Rieta, F. Castells, C. Sánchez, V. Zarzoso, and J. Millet, "Atrial activity extraction for atrial fibrillation analysis using blind source separation," IEEE Transactions on Biomedical Engineering, vol. 51, no. 7, pp. 1176-1186, 2004.

[4] L. De Lathauwer, "Blind separation of exponential polynomials and the decomposition of a tensor in rank- $\left(l_{r}, l_{r}, 1\right)$ terms," SIAM Journal on Matrix Analysis and Applications, vol. 32, no. 4, pp. 1451-1474, 2011.

[5] V. Zarzoso, "Parameter estimation in block term decomposition for noninvasive atrial fibrillation analysis," in IEEE 7th Int. Workshop Comput. Adv. Multi-Sensor Adapt. Process. (CAMSAP), Curaçao, Dutch Antilles, Dec. 2017.

[6] P. M. R. de Oliveira and V. Zarzoso, "Block term decomposition of ECG recordings for atrial fibrillation analysis: Temporal and inter-patient variability," Journal of Communication and Information Systems, vol. 34, pp. 111-119, 2019.

[7] P. Kirchhof and H. Calkins, "Catheter ablation in patients with persistent atrial fibrillation," European Heart Journal, vol. 38, no. 1, pp. 20-26, Jul. 2016.

[8] M. Haïssaguerre, P. Jaïs, D. C. Shah, A. Takahashi, M. Hocini, et al., "Spontaneous initiation of atrial fibrillation by ectopic beats originating in the pulmonary veins," New England Journal of Medicine, vol. 339, no. 10, pp. 659-666, 1998.

[9] J. Seitz, C. Bars, G. Théodore, S. Beurtheret, et al., "AF ablation guided by spatiotemporal electrogram dispersion without pulmonary vein isolation: A wholly patient-tailored approach," Journal of the American College of Cardiology, vol. 69, no. 3, pp. 303-321, 2017

[10] J. H. de M. Goulart, P. M. R. de Oliveira, R. C. Farias, V. Zarzoso, and P. Comon, "Alternating group lasso for block-term tensor decomposition and application to ECG source separation," IEEE Transactions on Signal Processing, vol. 68, pp. 26822696, 2020.

[11] M. Stridh and L. Sörnmo, "Spatiotemporal QRST cancellation techniques for analysis of atrial fibrillation," IEEE Transactions on Biomedical Engineering, vol. 48, no. 1, pp. 105-111, 2001.

[12] M. Yuan and Y. Lin, "Model selection and estimation in regression with grouped variables," Journal of the Royal Statistical Society: Series B (Statistical Methodology), vol. 68, no. 1, pp. 4967, 2006.

[13] J. A. Cadzow, "Signal enhancement-a composite property mapping algorithm," IEEE Transactions on Acoustics, Speech, and Signal Processing, vol. 36, no. 1, pp. 49-62, 1988.

[14] P. M. R. de Oliveira and V. Zarzoso, "Source analysis and selection using block term decomposition in atrial fibrillation," in 14th International Conference on Latent Variable Analysis and Signal Separation (LVA/ICA), Guildford, UK, Jul. 2018, pp. 46-56.

[15] V. Zarzoso and P. Comon, "Robust independent component analysis by iterative maximization of the kurtosis contrast with algebraic optimal step size," IEEE Transactions on Neural Networks, vol. 21, no. 2, pp. 248-261, 2010.

Address for correspondence:

Pedro Marinho R. de Oliveira

Les Algorithmes, Euclide B, 2000 route des lucioles, 06900 Sophia Antipolis Cedex, France marinho@i3s.unice.fr 